\title{
FUNDAMENTAL THEORY OF CONTINGENT DIFFERENTIAL EQUATIONS IN BANACH SPACE
}

\author{
BY \\ SHUI-NEE CHOW(1) AND J. D SCHUUR
}

\begin{abstract}
For a contingent differential equation that takes values in the closed, convex, nonempty subsets of a Banach space $E$, we prove an existence theorem and we investigate the extendability of solutions and the closedness and continuity properties of solution funnels. We consider first a space $E$ that is separable and reflexive and then a space $E$ with a separable second dual space. We also consider the special case of a pointvalued or ordinary differential equation.
\end{abstract}

0. Introduction. Consider the contingent differential equation

$$
D x \subset F(t, x)
$$

where $F$ maps $R \times E$ into the closed, convex, nonempty subsets of $E, E$ a Banach space. A solution to (1) is a function $\varphi$ mapping some interval $I$ into $E$ such that if $D \varphi(t)$ is the contingent derivative of $\varphi$, then $D \varphi(t) \subset F(t, \varphi(t))$ on $I$. In this paper we prove an existence theorem for the initial value problem associated with (1); we discuss the extendability of solutions and the closedness and continuity properties of solution funnels; and we investigate the initial value problem associated with (1) in the special case where $F$ is point-valued, i.e. when (1) is any ordinary differential equation.

In $\S 1$ we state basic definitions, we state the conditions to be placed on $F$ in the hypotheses of the existence theorem, and we give a characterization of solutions.

In particular, if $\left(t_{0}, x_{0}\right) \in R \times E$ is our initial point and $N$ is a neighborhood of $\left(t_{0}, x_{0}\right)$, then we assume that for all $(t, x) \in N, F(t, x)$ lies in a fixed bounded set and $F(t, x)$ is upper semicontinuous in a certain sense (stated in condition A).

Condition $\mathrm{A}$ is interesting in that it extends to Banach spaces Cesari's condition $Q$ [2] and it is similar to Marchaud's concept of regularity [11] and Zaremba's idea of upper semicontinuity [17]. And in the case $F$ is point-valued, condition A reduces to weak continuity.

In $\$ 2$ we prove our existence theorem in the case of $E$ a reflexive and separable space. In this case and under the above mentioned hypotheses we show that the initial value problem associated with (1) has a solution $\varphi(t)$. Further, the

Presented to the Society, January 17, 1972; received by the editors March 3, 1972.

AMS (MOS) subject classifications (1970). Primary 34A10, 34G05; Secondary 26A15.

Key words and phrases. Ordinary differential equations, contingent differential equations, existence of solutions, fundamental theory, Banach spaces, weak topology, upper semicontinuity.

(') Partially supported by the National Science Foundation under Contract NSF GU-2648. 
Ważewski result [15] holds: The strong derivative of $\varphi, \varphi^{\prime}$, exists and $\varphi^{\prime}(t)$ $\in F(t, \varphi(t))$ a.e. (strong or weak refers to limits in the strong or weak topology on $E)$.

Also, we discuss the extendability of solutions (using ideas of Corduneanu [5]), we prove that funnels of solutions are closed, and we discuss the continuity properties of solution funnels.

In $\S 3$ we investigate the initial value problem for $E$ a general Banach space. Consider the ordinary differential equation.

$$
x^{\prime}=f(t, x)
$$

where $f: R \times E \rightarrow E$.

It was shown by example in [6] and [16] that if we only assume $f$ is continuous, then the initial value problem for (2) need not necessarily have a solution. (In the special case of $E=E^{n}$, the continuity of $f$ does, of course, imply the existence of such a solution.)

Subsequently, existence theorems for the initial value problem for (2) were proved where, in addition to the assumption of the continuity of $f$, it was assumed:

$f=f_{1}+f_{2}$ where $f_{1}$ is completely continuous and $f_{2}$ satisfies a Lipschitz condition [9];

$f$ is uniformly continuous and its range lies in a compact set [5]; and

$f$ satisfies a Kamke-type condition [13].

In [1] the idea of a weak solution (i.e. a strongly continuous function whose weak derivative satisfies (1)) was used, and it was shown that if $f$ is weakly continuous and bounded and if $E$ is reflexive and separable then there exists a solution to the initial value problem.

In $\$ 3$ we prove the following: Let $E$ be embedded in its second dual space $E^{* *}$, which is assumed to be separable, and let $E^{* *}$ with the weak star topology be denoted by $E_{w}^{* *}$. If, in a neighborhood of $\left(t_{0}, x_{0}\right), f$ can be extended so that $f: R \times E^{* *} \rightarrow E^{* *}$ is continuous and $f$ is bounded in the strong norm, then there is a function $\varphi:\left(t_{0}-\delta, t_{0}+\delta\right) \rightarrow E^{* *}$ with $\varphi\left(t_{0}\right)=x_{0}$ which is strongly continuous and whose weak* derivative satisfies (2). If, additionally, $E$ is reflexive and its dual space is separable, then $\varphi$ has a strong derivative which satisfies (2) a.e.

In $\S 3$ we also investigate (1) when $E$ is a general Banach space. We show that $F$ can be defined as a mapping from $R \times E^{* *}$ into the closed, convex, nonempty subsets of $E^{* *}$ in such a way that condition A holds. Then assuming the range of $F$ lies in a fixed bounded set we prove that (1) has a solution $\varphi: R \rightarrow E^{* *}$. When $E$ is reflexive and $E^{*}$ separable we are back to the setting of $\S 2$.

Some of these results were presented in [4]. An existence theorem for ordinary differential equations under similar conditions is contained in [3].

1. Definitions and basic theorems. Let $E$ be a real Banach space with norm $\|\cdot\|$. Denote $E$, when equipped with the weak topology, by $E_{w}$ and denote the dual space of $E$ by $E^{*}$. 
Let $W$ be an open connected set in $R \times E$. Points in $W$ are denoted by $P$, $\left(t_{P}, x_{P}\right)$, or just $(t, x)$. For $P, Q \in W,\|P-Q\|=\max \left(\left|t_{P}-t_{Q}\right|,\left\|x_{P}-x_{Q}\right\|\right)$.

For $A \subset E, \overline{\mathrm{co}} A$ is the closure of the convex hull of $A$. And $\operatorname{cf}(E)$ is the collection of all nonempty, convex, closed subsets of $E$.

Definition 1. A function $f: W \rightarrow \operatorname{cf}(E)$ is said to satisfy condition A if there exists a countable set $\exists=\left\{F_{n} \in E^{*}\right\}$ such that, at each $P_{0} \in W$,

$$
f\left(P_{0}\right)=\bigcap_{n} Q_{n}\left[f\left(P_{0}\right)\right]
$$

where

$$
Q_{n}\left[f\left(P_{0}\right)\right]=\overline{\mathrm{co}} \cup\left\{f(P):\left|t_{P}-t_{0}\right|<1 / n,\left|F_{i}\left(x_{P}-x_{0}\right)\right|<1 / n,\right.
$$

$$
i=1,2, \ldots, n\}
$$

If $\Im$ is dense in $E^{*}$, the following hold.

(1) For $E=R^{n}$ condition A is equivalent to Cesari's condition $Q$ [2]. (This is also called semicontinuity in the sense of Cesari [10].)

(2) For $E=R^{n}$ and $f(P)$ is point-valued at each $P$, condition $\mathrm{A}$ is equivalent to the continuity of $f$.

(3) If $f(P)$ is point-valued at each $P$ and if we consider $W \subset R \times E_{w}$ and $f: W \rightarrow E_{w}$, then condition A is equivalent to the continuity of $f$. (We show this at the end of the section.)

If $\exists$ is not dense in $E^{*}$, but "smaller", then the set of solutions of our contingent equation will be larger.

Condition A yields directly the properties needed in $f(P)$ for an existence theorem and it avoids examining a topology on $\operatorname{cf}(E)$.

Definition 2. A set $A \subset W$ is an $\alpha$-set (Corduneanu [5]) if $A$ is bounded and if the $\inf \{\|P-Q\|: P \in A, Q \in \operatorname{Bdy}(W)\}>0$.

Definition 3. A mapping $f: W \rightarrow \operatorname{cf}(E)$ is said to satisfy condition B if for each $\alpha$-set $A \subset W$ there exists a constant $m$ such that $\|f(P)\| \leq m$ on $A$.

Definition 4. If $\left\{x_{n}\right\}$ is a sequence in $E, x_{n} \rightarrow x$ weakly means that for every $F$ in $E^{*}, F\left[x_{n}\right] \rightarrow F[x]$.

If $\varphi: I \rightarrow E$ ( $I$ an open interval in $R), \varphi(t)$ is weakly continuous means that for every $F$ in $E^{*}, F[\varphi(t)]$ is a continuous function of $t$.

When we say that a property holds nearly everywhere on $I$ we mean that it holds everywhere except, possibly, at a denumerable number of points.

Definition 5. Let $\Delta_{h} \varphi(t)=(\varphi(t+h)-\varphi(t)) h^{-1}$ and let

$$
D \varphi(t)=\left\{y \in E: \Delta_{h(n)} \varphi(t) \rightarrow y \text { weakly for some sequence } h(n) \rightarrow 0+\right\}
$$

A contingent differential equation is any expression of the form

$$
D x \subset f(t, x)
$$


where $f: W \rightarrow \operatorname{cf}(E)$.

A solution of (1) on $I$ is a continuous function $\varphi: I \rightarrow E$ such that $\varnothing \neq D \varphi(t)$ $\subset F(t, \varphi(t))$ nearly everywhere on $I$ (i.e. except, possibly, at a denumerable number of points).

Theorem 1. Let $\varphi: I \rightarrow E$ be continuous and assume that $D \varphi(t) \neq \varnothing$ nearly everywhere on I. Then $\varphi$ is a solution of (1) on $I$ if and only if for $t \in I$ and $m>0$ there exists an $\eta(t, m)>0$ such that

$$
0<h<\eta \Rightarrow \Delta_{h} \varphi(t) \in Q_{m}[f(t, \varphi(t))] .
$$

The following is used in the proof of Theorem 1.

Theorem 2. If $A$ is a closed convex set in $E$, if $\psi:(a, b) \rightarrow E$ is continuous, and if there exist sequences $\left\{y_{n} \in A\right\},\{h(n) \rightarrow 0+\}$ such that $\left[\Delta_{h(n)} \psi(t)-y_{n}\right] \rightarrow 0$ weakly, nearly everywhere on $(a, b)$, then

$$
\psi\left(t_{2}\right)-\psi\left(t_{1}\right) /\left(t_{2}-t_{1}\right) \in A \text { for } t_{1}, t_{2} \in(a, b), t_{1} \neq t_{2} .
$$

A proof of Theorem 2 when $E=E^{n}$ may be found in Zaremba [17], but for general Banach spaces we refer to Mlak [12].

Proof of Theorem 1. Let $\varphi(t)$, a solution of (1), and $t \in I, m>0$ be given. Choose $\eta>0$ such that $|s-t|<\eta$ implies

$$
\sup \left\{|s-t|:\left|F_{i}[\varphi(s)-\varphi(t)]\right|\right\}<1 / m \text { for } F_{i} \in \mathcal{F}, i=1,2, \ldots, m .
$$

Then $D \varphi(s) \subset F(s, \varphi(s)) \subset Q_{m}[f(t, \varphi(t))]$ nearly everywhere on $|s-t|<\eta$ implies $\Delta_{h} \varphi(t) \in Q_{m}[f(t, \varphi(t))]$ for $0<h<\eta$.

If $\Delta_{h} \varphi(t) \in Q_{m}[f(t, \varphi(t))]$ for $t \in I, h \in(0, \eta)$, then $D \varphi(t) \subset Q_{m}[f(t, \varphi(t))]$. But $m$ is arbitrary so

$$
D \varphi(t) \subset \bigcap_{n=1}^{\infty} Q_{n}[f(t, \varphi(t))]=f(t, \varphi(t)) .
$$

Corollary 1. In Theorem 2, and hence in Theorem 1, we may replace the phrase " $\varphi(t)$ is continuous and the stated conditions hold nearly everywhere" by " $\varphi(t)$ is absolutely continuous and the stated conditions hold almost everywhere" and the two theorems are again true.

Corollary 2. If $\left\{\varphi_{n}(t)\right\}$ is an equicontinuous sequence of solutions of (1) and if $\varphi_{n}(t) \rightarrow \varphi(t)$ weakly on $I$, then $\varphi(t)$ is a solution of $(1)$.

Proof. First, for $\epsilon>0$ there exists a $\delta$ such that $|h|<\delta$ implies $\| \varphi_{n}(t+h)$ $-\varphi_{n}(t) \|<\epsilon$ for all $n$ and $t \in I$. Then the weak limit lies in the same sphere, i.e., $\|\varphi(t+h)-\varphi(t)\|<\epsilon$, and $\varphi(t)$ is continuous.

Second, in the theorem we may choose $\eta(t, m)$ such that $0<h<\eta$ implies $\Delta_{h} \varphi_{n}(t) \in Q_{m}[f(t, \varphi(t))]$ for all $n$ sufficiently large. 
Third, $Q_{m}[f(t, \varphi(t))]$ is convex and closed, hence weakly closed, so $\Delta_{h} \varphi(t)$ $\in Q_{m}[f(t, \varphi(t))]$.

Addenda. (1) Relation of condition A to continuity: Assume that $f(P)$ is pointvalued at each $P \in W$ and consider $W \subset R \times E_{w}$. We shall show:

(i) $f$ satisfies condition $\mathrm{A} \Rightarrow f: W \rightarrow E_{w}$ is continuous;

(ii) $f: W \rightarrow E_{w}$ is continuous, $m=\sup \{\|P\|: P \in W\}<\infty$, and $\Im$ is dense in $E^{*} \Rightarrow f$ satisfies condition A.

Since $f\left(P_{0}\right)=\bigcap_{n=1}^{\infty} Q_{n}\left[f\left(P_{0}\right)\right]$ and $Q_{1}\left[f\left(P_{0}\right)\right] \supset Q_{2}\left[f\left(P_{0}\right)\right] \supset \ldots$, (i) follows.

Suppose $x_{0} \neq f\left(P_{0}\right)$. There exists a $G_{0} \in E^{*}$ such that $G_{0}\left(x_{0}-f\left(P_{0}\right)\right) \geq \eta$ and by the continuity of $f$ there is a weak neighborhood

$$
A=\bigcap_{i=1}^{M}\left\{Q:\left|t_{Q}-t_{0}\right|<\delta,\left|G_{i}\left(x_{Q}-x_{0}\right)\right|<\delta\right\},
$$

$G_{i} \in E^{*}$, of $P_{0}$ such that $f(A) \subset\left\{x: G_{0}\left(x-f\left(P_{0}\right)\right)<\eta\right\}$.

Choose $N$ such that $N>2 / \delta$ and such that for each $i \in[1, M]$ there is a $j \in[1, N]$ with $\left\|F_{j}-G_{i}\right\|<\delta / 4 m$ where $m=\sup \{\|P\|: P \in W\}$.

Then $\left|F_{j}\left(x_{Q}-x_{0}\right)\right|<1 / N(j \in[1, N])$ implies $\left|G_{i}\left(x_{Q}-x_{0}\right)\right| \leq\left|F_{j}\left(x_{Q}-x_{0}\right)\right|$ $+\left\|F_{i}-G_{i}\right\|\left\|x_{O}-x_{0}\right\|<\delta$ for $i \in[1, M]$ and hence

$$
Q_{N}\left[f\left(P_{0}\right)\right] \subset\left\{x: G_{0}\left(x-f\left(P_{0}\right)\right)<\eta\right\} .
$$

(2) A simple example to emphasize the difference between strong and weak continuity:

Let $E=l^{2}$ and let $e_{n}$ be the element of $E$ with one in the $n$th place and zeros elsewhere. Define $g$ by

$$
\begin{aligned}
g(t) & =0, \quad t \leq 0, \\
& =e_{1}, \quad t \geq 1, \\
& =[1 / n-1 /(n+1)]^{-1}\left\{e_{n} \cdot[t-1 /(n+1)]+e_{n+1} \cdot[1 / n-t]\right\}, \\
& t \in[1 /(n+1), 1 / n] .
\end{aligned}
$$

Then $g(1 / n)=e_{n}$ and $g(t)$ is continuous in the weak topology. But $g(t)$ is not continuous in the strong topology at $t=0$.

Now define $f: E \rightarrow E$ by $f(x)=g\left(\left\langle e_{1}, x\right\rangle\right)$, where $\langle\cdot, \cdot\rangle$ denotes the inner product in $E$. Thus $f$ is continuous in the weak topology but not in the strong topology.

2. Existence theorem and fundamental properties of solutions. In this section we assume, additionally, that $E$ is separable and reflexive.

Theorem 3. Let (1) be given and assume that $f(t, x)$ satisfies conditions $\mathrm{A}$ and $\mathrm{B}$. Then for $\left(t_{0}, x_{0}\right) \in W$ there exists an interval I containing $t_{0}$ and a solution $\varphi(t)$ of (1) on I such that $\varphi\left(t_{0}\right)=x_{0}$. Further, $\varphi^{\prime}(t)$ (the strong limit of $\Delta_{h} \varphi(t)$ as $\left.h \rightarrow 0\right)$ exists and $\varphi^{\prime}(t) \in f(t, \varphi(t))$ almost everywhere on $I$. 
Proof. Choose an $\alpha$-set $A \subset W$ such that $P_{0}=\left(t_{0}, x_{0}\right)$ is in the interior of $A$ and let $m$ be the constant given by condition B. Choose $a>t_{0}$ such that

$$
R=\left\{(t, x):\left|t-t_{0}\right| \leq a-t_{0},\left\|x-x_{0}\right\| \leq m\left(a-t_{0}\right)\right\} \subset A .
$$

With no loss of generality assume $t_{0} \leq t \leq a \leq t_{0}+1$.

Form a partition $\Delta_{n}$ of $\left[t_{0}, a\right]: t_{0}<t_{1}<\ldots<t_{n}=a$ with $\left(t_{i}-t_{i-1}\right) \leq 1 / n$ $(i=1, \ldots, n)$.

Define the polygonal line

$$
\begin{aligned}
\varphi_{n}\left(t_{0}\right) & =x_{0}, \\
\varphi_{n}(t) & =\varphi_{n}\left(t_{i-1}\right)+\left(t-t_{i-1}\right) v_{i-1} \quad \text { if } t_{i-1}<t \leq t_{i}(i=1, \ldots, n)
\end{aligned}
$$

where $v_{i-1} \in f\left(t_{i-1}, \varphi_{n}\left(t_{i-1}\right)\right)$ and $\left|v_{i-1}\right| \leq m$.

Since $\left\{\varphi_{n}(t)\right\}$ is a uniformly bounded, equicontinuous family, a subsequence $\left\{\varphi_{k}(t)\right\}$ converges weakly, uniformly on $\left[t_{0}, a\right]$, to a function $\varphi(t)$ on $\left[t_{0}, a\right]$. Then $\varphi(t) \in E$ for each $t, \varphi\left(t_{0}\right)=x_{0}$, and as in the proof of Theorem 1, Corollary 2, $\varphi(t)$ is continuous.

Let $m>0, t_{1} \in\left(t_{0}, a\right)$ be given. We claim there exists $N\left(m, t_{1}\right), \eta\left(m, t_{1}\right)>0$ such that $\varphi_{n}^{\prime}(t) \in Q_{m}\left[f\left(t_{1}, \varphi\left(t_{1}\right)\right)\right]$ for $\left|t-t_{1}\right|\langle\eta, n \geq N$ (' is here the right derivative in the strong sense). It will then follow from Theorem 2 that

$$
\left(\varphi_{n}\left(t_{3}\right)-\varphi_{n}\left(t_{2}\right)\right)\left(t_{3}-t_{2}\right)^{-1} \in Q_{m}\left[f\left(t_{1}, \varphi\left(t_{1}\right)\right)\right]
$$

for $n \geq N,\left|t_{i}-t_{1}\right|<\eta(i=2,3)$, hence by the convexity of $Q_{m}$ that

$$
\Delta_{h} \varphi\left(t_{1}\right) \in Q_{m}\left[f\left(t_{1}, \varphi\left(t_{1}\right)\right)\right]
$$

and thus, by Theorem 1 , that $\varphi(t)$ is a solution of $(1)$ on $\left[t_{0}, a\right]$.

To prove the claim assume $1 / m<\min \left(t_{1}-t_{0}, a-t_{1}\right)$ and choose $\eta \in(0$, $1 / 2 m)$ such that $\left|t-t_{1}\right| \leq 2 \eta$ implies $\left|F_{i}\left[\varphi_{n}(t)-\varphi_{n}\left(t_{1}\right)\right]\right|<1 / 2 m$ for every $n$ and $i=1, \ldots, m$.

Choose $N>2 / \eta$ such that $n \geq N$ implies $\left|F_{i}\left[\varphi_{n}\left(t_{1}\right)-\varphi\left(t_{1}\right)\right]\right|<1 / 2 m$, $i$ $=1, \ldots, M$. Then $\left|t-t_{1}\right| \leq \eta, n \geq N$, implies $\varphi_{n}^{\prime}(t)=v_{j} \in f\left(t_{j}, \varphi_{n}\left(t_{j}\right)\right)$ for $t_{j}$ a point of the subdivision $\Delta_{n}$ and further $\left|t-t_{j}\right|<\eta / 2$. Then $\left|t_{1}-t_{j}\right|<2 \eta$ so

$$
\begin{gathered}
\left|F_{i}\left[\varphi_{n}\left(t_{1}\right)-\varphi_{n}\left(t_{j}\right)\right]\right|<1 / 2 m \quad(i=1, \ldots, m), \\
\max \left\{\left|t_{j}-t_{1}\right|,\left|F_{i}\left[\varphi\left(t_{j}\right)-\varphi\left(t_{1}\right)\right]\right|(i=1, \ldots, m)\right\}<1 / m,
\end{gathered}
$$

and $\varphi_{n}^{\prime}(t) \in Q_{m}\left[f\left(t_{1}, \varphi\left(t_{1}\right)\right)\right]$.

To see that $\varphi^{\prime}(t)$ exists a.e. and hence that $\varphi^{\prime}(t) \in D \varphi(t) \subset f(t, \varphi(t))$ a.e. on $I$ we employ a theorem of Pettis [14] which states: For a reflexive space $E$, a function of bounded variation $\psi: I \rightarrow E$ is strongly differentiable a.e. and its derivative is integrable in the sense of Bochner. 
From the definition of $\varphi_{n}(t),\left\|\varphi_{n}\left(t_{2}\right)-\varphi_{n}\left(t_{1}\right)\right\| \leq m\left|t_{2}-t_{1}\right|\left(t_{2}, t_{1} \in I\right)$. Hence, $\left\|\varphi\left(t_{2}\right)-\varphi\left(t_{1}\right)\right\| \leq m\left|t_{2}-t_{1}\right|$ and $\varphi$ is of bounded variation.

Remark. In Theorem 3 we may weaken condition B as follows: For each $\alpha$-set $A \subset W$ there exists a constant $m>0$ such that $f(P) \cap\{x:\|x\| \leq m\} \neq \varnothing$ on A.

We now state some fundamental properties of solutions of (1). We sketch only a few proofs since they involve standard techniques from the theory of ordinary differential equations.

Definition 6. Let $\varphi(t)$ be a solution of (1) passing through $\left(t_{0}, x_{0}\right)$. Let $\left(\alpha_{\varphi}, \omega_{\varphi}\right)$ be the domain of $\varphi$ and $\Gamma_{\varphi}^{+}=\left\{(t, \varphi(t)): t_{0} \leq t<\omega_{\varphi}\right\}$. Then $\psi(t)$ is a right extension of $\varphi(t)$, and $\varphi(t)$ is extendable to the right if (i) $\psi$ is a solution of (1) passing through $\left(t_{0}, x_{0}\right)$; and (ii) $\omega_{\varphi}<\omega_{\psi}, \varphi(t)=\psi(t)$ on $\left[t_{0}, \omega_{\varphi}\right)$. If $\varphi$ is not extendable to the right, then $\varphi$ is fully extended to the right .

Theorem 4. The solution $\varphi(t)$ of (1) is extendable to the right if and only if $\Gamma_{\varphi}^{+}$is an $\alpha$-set. Further, each solution of (1) which is not fully extended to the right has a right extension which is fully extended to the right.

Proof. See Corduneanu [5].

We may similarly discuss left extensions and extensions of solutions.

Definition 7. For $P \in W$, let $\Phi(P)$ be the family of all solutions of (1) passing through $P$. If all members of $\Phi(P)$ are defined on $[\gamma, \delta]$, then $Z(P ; \gamma, \delta)$ or simply $Z(P)=\{(t, \varphi(t)): \gamma \leq t \leq \delta, \varphi \in \Phi(P)\}$.

For $A \subset W$, assume all members of $\Phi(P)$ are defined on $[\gamma, \delta]$ for each $P \in A$. Then $Z(A)=\bigcup\{Z(P): P \in A\}$.

Theorem 5. If $A \subset W$ is closed and bounded and if all solutions of (1) through any point of $A$ are defined on $[\gamma, \delta]$, then $Z(A)$ is an $\alpha$-set and is closed.

Proof. We sketch the proof. First assume $A$ is a point $P$. Using weak compactness we can extend the proof to sets.

If $\left\{\varphi_{n}(t, P)\right\}$ is a sequence of solutions of (1) passing through $P$ whose graphs lie in an $\alpha$-set $B$ for $t \in I \subset[\gamma, \delta]$, then $\left\{\varphi_{n}(t, P)\right\}$ is a uniformly bounded, equicontinuous family. Hence by Corollary 2 of Theorem 1 some subsequence of $\left\{\varphi_{n}(t, P)\right\}$ converges weakly to a solution of (1) on $I$.

Also, such an $\alpha$-set $B$ always exists, viz., the usual small rectangle with center at $P$.

Now if $Z(P)$ is not an $\alpha$-set, then there exists a sequence $Q_{n}=\left(t_{n}, \varphi_{n}\left(t_{n}, P\right)\right)$ $\in Z(P)$ such that $t_{n} \rightarrow t_{0} \in[\gamma, \delta]$ and either $\left\|\varphi_{n}\left(t_{n}, P\right)\right\| \rightarrow \infty$ (as a limit) or $Q_{n} \rightarrow \operatorname{Bdy}(W)$ as $t_{n} \rightarrow t_{0}$. By standard methods we may find a subsequence $\varphi_{k}(t, P) \rightarrow \varphi(t, P)$ weakly where $\varphi(t, P)$ is a solution of (1) through $P$ which cannot be defined at $t=t_{0}$. This gives a contradiction.

If $Z(P)$ is an $\alpha$-set, then $\|f(t, x)\| \leq m$ on $Z(P)$ and $\Phi(P)$, the family of solutions of (1) passing through $P$, is equicontinuous. The closedness of $Z(P)$ again follows from Theorem 1, Corollary 2. 
Theorem 6. Let $\left\{A_{n}\right\}$ be a sequence of closed bounded sets in $W$ with $A_{1} \supset A_{2}$ $\supset$... and assume that all solutions of (1) passing through any point of $A_{1}$ are defined on $[\gamma, \delta]$. Then $\bigcap_{n=1}^{\infty} Z\left(A_{n}\right)=Z\left(\cap_{n=1}^{\infty} A_{n}\right)$.

Proof. Suppose $Q \in \cap_{n=1}^{\infty} Z\left(A_{n}\right)$. Then $Q=\left(t_{Q}, \varphi_{n}\left(t_{Q}, P_{n}\right)\right)$ where $\varphi_{n}$ is a solution of (1) and $P_{n} \in A_{n}$. Now $\left\{\varphi_{n}\left(t, P_{n}\right)\right\}$ is an equicontinuous family since $Z\left(A_{1}\right)$ is an $\alpha$-set. So some subsequence of $\left\{\varphi_{n}\left(t, P_{n}\right)\right\}$ converges weakly, uniformly on $[\gamma, \delta]$, to $\varphi\left(t_{0}, P_{0}\right)$. Then $P_{0} \in \cap_{n=1}^{\infty} A_{n}$ and $Q=\left(t_{Q}, \varphi\left(t_{Q}, P_{0}\right)\right)$.

The other half of the proof is immediate.

Specific continuity properties of solutions follow from Theorem 6. For example:

Corollary 1. Let $\left\|P_{n}-P_{0}\right\| \rightarrow 0$ as $n \rightarrow \infty\left(P_{n}, P_{0} \in W\right)$ and let $\varphi_{n}(t)$ be a fully extended solution of (1) through $P_{n}$ with $\left(\alpha_{n}, \omega_{n}\right)$ as its domain of definition, $n=0,1, \ldots$ Then

$$
\lim \sup \alpha_{n} \leq \alpha_{0}<\omega_{0} \leq \lim \inf \omega_{n}
$$

Corollary 2. For $P \in W$ let $Z_{t}(P)=Z(P) \cap\{(s, x): s=t, x \in E\}$. Let $\| P_{n}$ $-P_{0} \| \rightarrow 0$ as $n \rightarrow \infty\left(P_{n}, P_{0} \in W\right)$ and assume $Z_{t}\left(P_{0}\right) \neq \varnothing$. Then given $\epsilon>0$, $F \in E^{*}$ with $F(x) \leq 0$ for all $x \in Z_{t}\left(P_{0}\right)$, there exists an $N$ such that $n \geq N$ implies $F(y) \leq \epsilon$ for all $y \in Z_{t}\left(P_{n}\right)$.

3. Differential equations in a nonreflexive Banach space. In this section we again consider the initial value problem. We drop the requirement that $E$ be reflexive but we do require that the second dual space of $E$ be separable. Hence the dual space of $E$ and $E$ itself are separable. First we make some remarks on ordinary differential equations.

Consider the initial value problem

$$
x^{\prime}=f(t, x), \quad x(0)=0,
$$

where $f: R \times E \rightarrow E$ is point-valued.

As the example of Dieudonné [6] shows, even if $f$ is uniformly continuous a solution of (1) may not exist in $E$. But one does exist in $E^{* *}$, the second dual space of $E$, and in fact this is also true in general.

Let $E$ be embedded in $E^{* *}$. On $E^{* *}$ we shall use both the norm $\|\cdot\|$ topology and the weak* topology, i.e. the topology induced by the functionals in $E^{*}$ considered as a subset of $E^{* * *}$. We shall denote $E^{* *}$, when equipped with the weak* topology, by $E_{w}^{* *}$.

Let $I=[-1,1], W=\left\{(t, x) \in R \times E^{* *}:|t| \leq 1,\|x\| \leq 1\right\}$, and $\mathcal{B}=\{x(\cdot): I$ $\rightarrow E^{* *}: x(t)$ is continuous $\},\|x(\cdot)\|=\sup _{I}\|x(t)\|$. Since $E^{* *}$ is separable, $\mathcal{B}$ is separable. 
For $\epsilon>0, \quad F \in E^{*}, x(\cdot) \in \mathcal{B}$, let $N_{\epsilon, F}[x(\cdot)]=\{y(\cdot) \in \mathcal{B}:|F[y(t)-x(t)]|$ $<\epsilon$ on $I\}$. Using these sets and all finite intersections of these sets we have a base for a weak topology on $\mathcal{B}$ and we denote $\mathcal{B}$ with this topology by $\mathcal{B}_{w}$. Since $\mathcal{B}$ is separable, $\mathcal{B}_{w}$ is separable and since $E^{*}$ is separable, $\mathcal{B}_{w}$ satisfies the second axiom of countability. Hence, in $\mathcal{B}_{w}$, sequential compactness will imply compactness.

Let

$$
\mathcal{E}=\{x(\cdot) \in \mathcal{B}: x(0)=0,\|x(\cdot)\| \leq 1 \text { and }\|x(t)-x(s)\| \leq|t-s|\} .
$$

If $\left\{x_{n}(\cdot)\right\}$ is a sequence in $\varepsilon$, then $\left\{x_{n}(\cdot)\right\}$ is uniformly bounded. And since the unit sphere in $E^{* *}$ is weak* compact we may apply Ascoli's theorem to obtain a subsequence $\left\{x_{k}(\cdot)\right\}$ and an $x_{0}(\cdot)$ such that $F\left[x_{k}(t)-x_{0}(t)\right] \rightarrow 0$ for every $F \in E^{*}$ uniformly in $t$.

Now $x_{k}(t) \rightarrow x_{0}(t)$ in $E_{w}^{* *}$ implies $\left\|x_{0}(t)\right\| \leq \varliminf_{n}\left\|x_{n}(t)\right\|$. Hence $\left\|x_{0}(\cdot)\right\| \leq 1$, $\left\|x_{0}(t)-x_{0}(s)\right\| \leq|t-s|$, and $x_{0}(0)=0$ so $x_{0}(\cdot) \in \varepsilon$ and $\varepsilon$ is a compact set in $\mathcal{B}_{w}$.

Assume that $f: W \cap(R \times E) \rightarrow E$ can be extended to a function $f: W$ $\rightarrow E^{* *}$ such that:

(i) $\|f(t, x)\|$ is bounded on $W$. We denote the bound by $\|f\|$ and for simplicity we assume $\|f\| \leq 1$.

(ii) $f: W_{w} \rightarrow E_{w}^{* *}$ is continuous and it is uniformly continuous in $x$. By $W_{w}$ we mean $W$ with the $R \times E_{w}$ topology and by uniform continuity in $x$ we mean that given $\epsilon>0, F \in E^{*}$, there exists a weak* neighborhood $M(x)$ such that, for all $\|x\| \leq 1, y \in M(x)$ implies $|F[f(t, y)-f(t, x)]|<\epsilon$ for all $t \in I$.

Define $T: E^{*} \times I \times \mathcal{E} \rightarrow R$ by

$$
T(F, t, x(\cdot))=\int_{0}^{t} F[f(s, x(s))] d s .
$$

Then (i) $T$ is linear in $F$ and $|T(F, t, x(\cdot))| \leq\|F\||t|\|f\|$;

(ii) $\left|T\left(F, t_{2}, x(\cdot)\right)-T\left(F, t_{1}, x(\cdot)\right)\right| \leq\|F\|\left|t_{2}-t_{1}\right|\|f\|$;

(iii) for $\epsilon>0, F \in E^{*}, x_{1}(\cdot) \in \mathcal{E}$, there is a neighborhood $N_{\epsilon, F}\left[x_{1}(\cdot)\right]$ such that $x_{2}(\cdot) \in N_{\epsilon, F}\left[x_{1}(\cdot)\right]$ implies $\left|T\left(F, t, x_{2}(\cdot)\right)-T\left(F, t, x_{1}(\cdot)\right)\right|<\epsilon|t|$.

Fix $x(\cdot) \in \mathcal{E}$ and $t \in I$ and let $\Phi(t, x(\cdot)) \in E^{* *}$ be the bounded linear functional defined by $T$. This, in fact, is the Dunford third integral [7]. Then

(iv) $\|\Phi(t, x(\cdot))\| \leq|t|\|f\|$;

(v) $\left\|\Phi\left(t_{2}, x(\cdot)\right)-\Phi\left(t_{1}, x(\cdot)\right)\right\| \leq\left|t_{2}-t_{1}\right|\|f\|$.

Let $y(t)=\Phi(t, x(\cdot))$. Then

(vi) $\|y(t)\| \leq 1$ for $t \in I$ and $y(0)=0$;

(vii) $\left\|y\left(t_{2}\right)-y\left(t_{1}\right)\right\| \leq\left|t_{2}-t_{1}\right|$;

(viii) for $\epsilon>0, F \in E^{*}, x_{1}(\cdot) \in \varepsilon$ there is a neighborhood $N_{\epsilon, F}\left[x_{1}(\cdot)\right]$ such that $x_{2}(\cdot) \in N_{\epsilon, F}\left[x_{1}(\cdot)\right]$ implies $\left|F\left[y_{2}(\cdot)-y_{1}(\cdot)\right]\right|<\epsilon$.

We thus have a mapping $\supset: \varepsilon \rightarrow \varepsilon$ where $\supset$ is continuous in the $\mathcal{B}_{w}$ topology and we can use the Schauder-Tychonov fixed point theorem [8, p. 405] to infer the existence of an $x(t) \in \mathcal{E}$ (i.e. $x(0)=0,\|x(t)\| \leq 1$, and $\|x(t)-x(s)\|$ $\leq|t-s|)$ such that 


$$
x(t)=\int_{0}^{t} F[f(s, x(s))] d s \text { for all } F \in E^{*} .
$$

Further, if $F \in E^{*}$ and $\epsilon>0$ then there exists a $\delta$ such that $|h|<\delta$ implies

$$
\left|F\left[\frac{x(t+h)-x(t)}{h}-f(t, x(t))\right]\right|=\left|\frac{1}{h} \int_{t}^{t+h} F[f(s, x(s))-f(t, x(t))] d s\right|<\epsilon
$$

i.e., the weak derivative of $x(t), \dot{x}(t)$, exists and $\dot{x}(t)=f(t, x(t))$.

We have thus proved

Theorem 7. Let (2) be given and assume that in a neighborhood $W$ of the origin $f$ can be extended to a function mapping $R \times E^{* *}$ into $E^{* *}$ in such a way that

(i) $\|f(t, x)\|$ is bounded on $W$; and

(ii) $f(t, x)$ is weakly continuous in $(t, x)$ and is uniformly weakly continuous in $x$.

Then there exists a function $x(t)$, with values in $E^{* *}$, such that $x(0)=0,\|x(t)\|$ is Lipschitz continuous, and the weak derivative of $x(t), \dot{x}(t)$, exists and $\dot{x}(t)=f(t, x(t))$.

We can also give an existence theorem for the contingent differential equation (1) in the case where $E$ is not reflexive, but $E^{* *}$ is separable.

We first observe that if $f: R \times E \rightarrow \operatorname{cf}(E)$ satisfies condition $A$, then $f$ can be extended to a function $\hat{f}: R \times E^{* *} \rightarrow \operatorname{cf}\left(E^{* *}\right)$ where $\hat{f}$ satisfies condition A (with respect to $\Im$ which is considered as a subset of $\left.E^{* * *}\right)$.

For $P \in E^{* *}$ let

$$
\hat{f}(P)=\bigcap_{n=1}^{\infty} \overline{\mathrm{co}} \cup\left\{f(Q):\left|t_{Q}-t_{P}\right|<1 / n,\left|F_{i}\left[x_{Q}-x_{P}\right]\right|<1 / n,\right.
$$

$$
i=1, \ldots, n\}
$$

where $Q \in E \subset E^{* *}$ and $F_{1}, F_{2}, \ldots \in \beth$.

Assume $f: R \times E \rightarrow \operatorname{cf}(E)$ satisfies condition A. Then

(i) $\hat{f}: R \times E^{* *} \rightarrow \operatorname{cf}\left(E^{* *}\right)$ and if $P \in E, \hat{f}(P)=f(P)$.

(ii) $\hat{f}$ satisfies condition $\mathrm{A}$.

To see (ii), we note that (a)

$$
\begin{aligned}
& \hat{f}\left(P_{0}\right)=\bigcap_{n=1}^{\infty} \overline{\mathrm{co}} \cup\left\{f(P):\left|t_{P}-t_{P_{0}}\right|<1 / n,\left|F_{i}\left[x_{P}-x_{P_{0}}\right]\right|<1 / n,\right. \\
& i=1, \ldots, n\} \\
& \subset \bigcap_{n=1}^{\infty} \overline{\mathrm{co}} \cup\left\{\hat{f}(P):\left|t_{P}-t_{P_{0}}\right|<1 / n,\left|F_{i}\left[x_{P}-x_{P_{0}}\right]\right|<1 / n,\right. \\
& i=1, \ldots, n\}
\end{aligned}
$$

(b) for $n$ fixed, we have for $m \geq n$ 


$$
\begin{aligned}
& R_{n}\left[\hat{f}\left(P_{0}\right)\right]=\bigcup\left\{\hat{f}(P):\left|t_{P}-t_{P_{0}}\right|<1 / n,\left|F_{i}\left[x_{P}-x_{P_{0}}\right]\right|<1 / n,\right. \\
& i=1,2, \ldots, n\} \\
& \subset \cup\left\{\overline{\mathrm{co}} \cup\left\{f(Q):\left|t_{Q}-t_{P}\right|<1 / m,\left|F_{i}\left[x_{Q}-x_{P}\right]\right|<1 / m, i=1, \ldots, m\right\}:\right. \\
& \left.\left|t_{P}-t_{P_{0}}\right|<1 / n,\left|F_{i}\left[x_{P}-x_{P_{0}}\right]\right|<1 / n, i=1,2, \ldots, n\right\} \\
& \subset \overline{\mathrm{co}} \cup\left\{f(Q):\left|t_{Q}-t_{P_{0}}\right|<1 / m+1 / n,\left|F_{i}\left[x_{Q}-x_{P_{0}}\right]\right|\right. \\
& <1 / m+1 / n, i=1,2, \ldots, n\} \text {. }
\end{aligned}
$$

Since this is true for every $m \geq n$, we have

$$
\begin{aligned}
Q_{n}\left[\hat{f}\left(P_{0}\right)\right]=\overline{\operatorname{co}} R_{n}\left[\hat{f}\left(P_{0}\right)\right] & \\
\subset \overline{\operatorname{co}} \cup\left\{f(Q):\left|t_{Q}-t_{P_{0}}\right| \leq 1 / n,\left|F_{i}\left[x_{Q}-x_{P_{0}}\right]\right|\right. & \leq 1 / n, \\
i & =1,2, \ldots, n\} .
\end{aligned}
$$

Now if we take the intersection from $n=1$ to $n=\infty$ on the left and then on the right, we obtain

$$
\bigcap_{n=1}^{\infty} Q_{n}\left[\hat{f}\left(P_{0}\right)\right] \subset \hat{f}\left(P_{0}\right)
$$

and we have proved (ii).

By using the method in Theorem 3 we have the following:

Theorem 8. If $f$ satisfies condition $\mathrm{A}$ and if $\hat{f}$ is bounded on every $\alpha$-set, then the equation $D x \subset \hat{f}(t, x)$ has solution for the initial value problem.

Remark. For the continuity properties of solutions we need to know more about the set $\Im$, considered as a subset of $E^{* * *}$, and separability of $E^{*}$. This can then be discussed as in $\$ 2$.

\section{BiBLIOGRAPHY}

1. Ju. G. Borisovič, $A$ weak topology and periodic solutions of differential equations, Dokl. Akad. Nauk SSSR 136 (1961), 1269-1272 = Soviet Math. Dokl. 2 (1961), 176-179. MR 22 \#179.

2. L. Cesari, Existence theorems for weak and usual optimal solutions in Lagrange problems with unilateral constraints. I, II, Trans. Amer. Math. Soc. 124 (1966), 369-430. MR 34\#3392; \#3393.

3. S. N. Chow and J. D. Schuur, An existence theorem for ordinary differential equations in Banach spaces, Bull. Amer. Math. Soc. 77 (1971), 1018-1020.

4. Contingent differential equations in Banach spaces, Notices Amer. Math. Soc. 19 (1972), A-137. Abstract 691-34-41.

5. C. Corduneanu, Equazioni differenziali negli spazi di Banach, teoremi di esistenza e di prolungabilità, Atti Accad. Naz. Lincei Rend. Cl. Sci. Fis. Mat. Nat. (8) 23 (1957), 226-230. MR 20 \#3312.

6. J. Dieudonné, Deux exemples singuliers d'équations différentielles, Acta Sci. Math. Szeged 12 (1950), Leopoldo Fejér et Frederico Riesz LXX annos natis dedicatus, pars B, 38-40. MR 11, 729.

7. N. Dunford, Integration of abstract functions, Bull. Amer. Math. Soc. 42 (1937), 24; See also, B. 
J. Pettis, On integration in vector spaces, Trans. Amer. Math. Soc. 44 (1938), 277-304.

8. P. Hartman, Ordinary differential equations, Wiley, New York, 1964. MR 30 \# 1270.

9. M. A. Krasnosel' skiī and S. G. Kreìn, Nonlocal existence theorems and uniqueness theorems for systems of ordinary differential equations, Dokl. Akad. Nauk SSSR 102 (1955), 13-16. (Russian) MR $17,151$.

10. A. Lasota and C. Olech, On Cesari's semicontinuity condition for set valued mappings, Bull. Acad. Polon. Sci. Sér. Sci. Math. Astronom. Phys. 16 (1968), 711-716. MR 39 \#138.

11. A Marchaud, Sur les champs de demi-cônes et équations différentielles du premier ordre, Bull. Soc. Math. France 62 (1934), 1-38.

12. W. Mlak, Note on the mean value theorem, Ann. Polon. Math. 3 (1956), 29-31. MR 18, 724.

13. C. Olech, On the existence and uniqueness of solutions of an ordinary differential equation in the case of Banach space, Bull. Acad. Polon. Sci. Sér. Sci. Math. Astronom. Phys. 8 (1960), 667-673. MR 26 \# 5247.

14. B. J. Pettis, A note on regular Banach spaces, Bull. Amer. Math. Soc. 44 (1938), $420-428$.

15. T. Ważewski, Sur une condition équivalente à l'équation au contingent, Bull. Acad. Polon. Sci. Sér. Sci. Math. Astronom. Phys. 9 (1961), 865-867. MR 24 \# A3328.

16. J. A. Yorke, A continuous differential equation in Hilbert space without existence, Funkcial. Ekvac. 13 (1970), 19-21. MR 41 \# 8792.

17. S. Zaremba, Sur les équations au paratingent, Bull. Sci. Math. (2) 60 (1936), 139-160.

Department of Mathematics, Michigan State University, East Lansing, Michigan 48823 